\title{
Simulation of the Through-Focus Modulation Transfer Functions According to the Change of Spherical Aberration in Pseudophakic Eyes
}

\author{
Jae-hyung Kim', Myoung Joon Kim²*, Geunyoung Yoon ${ }^{3}$, Jae Yong Kim², and Hungwon Tchah ${ }^{2}$ \\ ${ }^{l}$ Department of Ophthalmology, Chungbuk National University College of Medicine, \\ Cheongju 362-711, Korea \\ ${ }^{2}$ Department of Ophthalmology, University of Ulsan College of Medicine, Asan Medical Center, \\ Seoul 138-736, Korea \\ ${ }^{3}$ Department of Ophthalmology and Center for Visual Science, University of Rochester, Rochester,
} NY 14642, USA

(Received December 26, 2014 : revised June 5, 2015 : accepted June 17, 2015)

\begin{abstract}
To evaluate the effects of spherical aberration (SA) correction on optical quality in pseudophakic eyes, we simulated the optical quality of the human eye by computation of the modulation transfer function (MTF). We reviewed the medical records of patients who underwent cataract surgery in Asan Medical Center, retrospectively. A Zywave aberrometer was used to measure optical aberrations at 1-12 postoperative months in patients with AR40e intraocular lens implants. The MTF was calculated for a $5 \mathrm{~mm}$ pupil from measured wavefront aberrations. The area under the MTF curve (aMTF) was analyzed and the maximal aMTF was calculated while changing the SA $(-0.2 \sim+0.2 \mu \mathrm{m})$ and the defocus $(-2.0$ $\sim+2.0$ D). Sixty-four eyes in 51 patients were examined. The maximal aMTF was $6.61 \pm 2.16$ at a defocus of $-0.25 \pm 0.66 \mathrm{D}$ with innate SA, and $7.64 \pm 2.63$ at a defocus of $0.08 \pm 0.53 \mathrm{D}$ when the SA was 0 (full correction of SA). With full SA correction, the aMTF increased in 47 eyes (73.4\%; Group 1) and decreased in 17 eyes (26.6\%; Group 2). There were statistically significant differences in $Z(3,-1)$ (vertical coma; $P=0.01$ ) and $\mathrm{Z}(4,4)$ (tetrafoil; $P=0.04$ ) between the groups. The maximal aMTF was obtained at an SA of $+0.01 \mu \mathrm{m}$ in Group 1 and an SA of $+0.13 \mu \mathrm{m}$ in Group 2. Optical quality can be improved by full correction of SA in most pseudophakic eyes. However, residual SA might provide benefits in eyes with significant radially asymmetric aberrations.
\end{abstract}
Keywords: Aspheric intraocular lens, Coma, Modulation transfer function, Spherical aberration, Tetrafoil, Simulation
OCIS codes : (170.1610) Clinical applications; (170.4470) Ophthalmology; (170.4460) Ophthalmic optics and devices

\section{INTRODUCTION}

Since the measurement of aberrations of the human eye using the Shack-Hartmann (SH) sensor was introduced twenty years ago, aberration has been widely used in ophthalmology. $[1,2]$ Nowadays, the implantation of an aspheric intraocular lens (IOL) has become popular after cataract surgery, as well as wavefront correction in refractive surgery [3, 4]. Theoretically, an aspheric IOL should improve retinal image quality by compensating corneal spherical aberration (SA), which is the major cause of decreasing image quality and is usually positive in eyes with cataracts [4-8]. However, there were some debates about the benefits of aspheric IOL use and also disagreements regarding the benefits of postoperative residual SA in patients with aspheric IOLs. Nowadays, commercially available aspheric IOLs have various SAs; hence IOLs with specific SAs can be targeted for individual patients [9-11]. However, there is no clear target SA for optimal visual outcome [12]. Some studies indicated that complete correction of SA improved visual performance, but some model-eye studies could not indicate best visual quality for a fully corrected SA [12-15]. Inter-subject variability

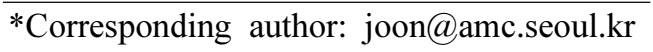


and interaction of the different sources of optical aberration may also make it difficult to determine the ideal SA of an IOL $[16,17]$.

A previous adaptive optics simulation study reported quantifiable results after full correction of SA [14]. However, no report has yet indicated how many patients achieved improved visual quality following full correction of SA or decreased visual quality. Therefore, we investigated what percentage of the patients would have visual improvement following full correction of SA (zero SA) and analyzed the optimal SA after cataract surgery.

\section{METHODS}

\subsection{Patient Characteristics}

Charts of patients who underwent phacoemulsification with implantation of spherical monofocal IOLs (AR40e, Abbott Medical Optics, Inc., Santa Ana, CA) at the Asan Medical Center from April 2007 to February 2010 were reviewed. All procedures were performed by the same surgeon (H. Tchah). Inclusion criteria were uneventful phacoemulsification with implantation of AR40e, and clinically well-positioned IOL in the capsular bag. Exclusion criteria were improper aberration data with less than $5 \mathrm{~mm}$ pupil size, post-operative decentered or tilted IOL, posterior capsular opacification (based on slit lamp examination) within the follow-up period, previous refractive surgery, or other corneal diseases.

\subsection{Manipulation of Higher-order Aberration}

HOAs were measured with Zywave ${ }^{\mathrm{TM}}$ (Bausch \& Lomb, Rochester, $\mathrm{NY}$ ) using the $\mathrm{SH}$ principle after using a mydriatic agent $\left(0.5 \%\right.$ tropicamide and $0.5 \%$ phenylephrine, Mydrin- $\mathrm{P}^{\mathbb{R}}$, Santen, Osaka, Japan). In brief, a polarized light source produced a small spot on the retina. Diffusely reflected light went through the lens and cornea and was relayed to the plane of the lenslet array. A CCD sensor recorded the spot array pattern and ocular aberration was reconstructed with the relative spot displacements (Fig. 1) [1, 2]. HOAs were normalized with a pupil size of $5 \mathrm{~mm}$. For computation of the MTF, the monochromatic PSF was computed by applying a Fourier transform to the pupil function using a program developed with MATLAB software (MathWorks,

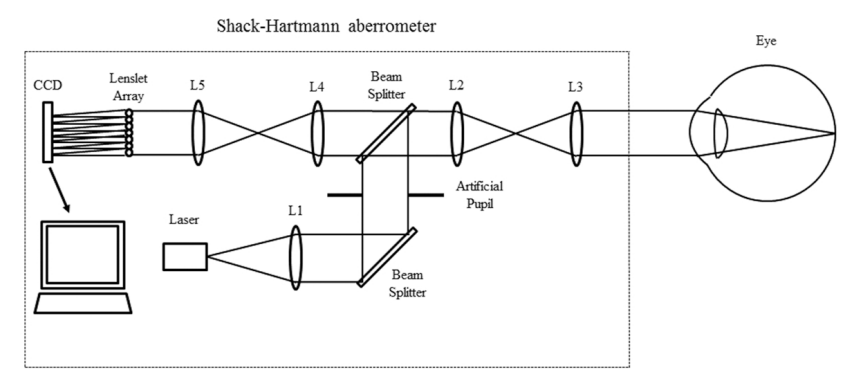

FIG. 1. Schematic diagram of the measurement of aberrations of the human eye using the SH sensor [2].

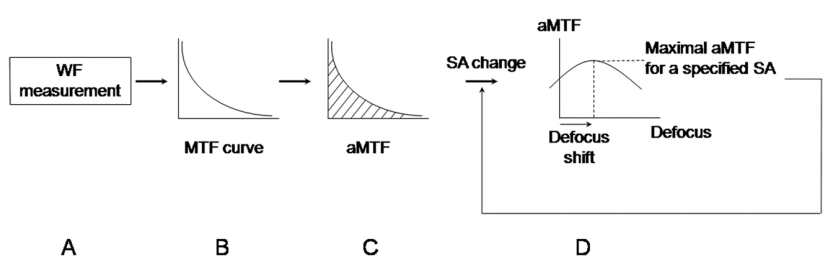

FIG. 2. Schematic illustration of the simulation of HOA to assess optical quality.

Natick, MA), as described previously [18]. The area under the MTF curve (aMTF) was the summation of MTFs according to spatial frequencies, which provided overall information on the ocular optical quality [19]. The aMTF was calculated up to $60 \mathrm{cpd}$ - the highest detectable spatial frequency for human eyes [20].

To simulate the correction of SA, Zernike coefficients of SA in each subject were modified from $-0.2 \mu \mathrm{m}$ to +0.2 $\mu \mathrm{m}$ with an interval of $0.01 \mu \mathrm{m}$. At each specific SA, through-focus aMTF were calculated with the range of defocus from $-2.0 \mathrm{D}$ to $+2.0 \mathrm{D}$ with an interval of $0.1 \mathrm{D}$. The maximal aMTF was the maximum value of the aMTF according to the changes of SA and defocus, which with the maximal aMTF were recorded (Fig. 2). The calculated aMTFs with innate SA and those after full correction of innate SA were analyzed. According to the differences between calculated aMTFs before and after full correction of innate SA, the total group was divided into two groups, eyes with increased aMTF (Group 1) and eyes with decreased aMTF (Group 2).

\subsection{Statistical Analysis}

All data were analyzed using SPSS version 14.0 software (SPSS Inc., Chicago, IL). Continuous variables were described as means and standard deviations, and compared using the Mann-Whitney U test and repeated ANOVA.[21] A $P$-value less than 0.05 was considered statistically significant.

\section{RESULTS}

We examined 64 eyes from 51 patients. Table 1 shows the demographic and postoperative refractive data. The average target spherical equivalent (SE) was $-1.02 \pm 1.21 \mathrm{D}$ and the average absolute prediction error (the difference between target SE and postoperative SE) was $0.16 \pm 0.47$ D. Full correction of innate SA increased the MTF at spatial frequencies less than $22 \mathrm{cpd}$ with statistically significant differences (Mann-Whitney U test, $p<0.05$; Fig. 3A). The average defocus required to obtain maximal aMTF $(6.61 \pm$ 2.16 with innate SA and $7.64 \pm 2.63$ after full correction of SA) was $-0.25 \pm 0.66$ diopter (D) and $0.08 \pm 0.53 \mathrm{D}$, respectively (Table 2, Fig. 3B).

Forty-seven eyes $(73.4 \%$ of 64 eyes) had increased aMTF after full correction of the SA (Group 1, Table 2). 
Simulation of the Through-Focus Modulation Transfer Functions According to - . - Jae-hyung Kim et al.

TABLE 1. Demographic characteristics and postoperative refractive errors and visual acuity

\begin{tabular}{l|c}
\hline \hline & Mean \pm Standard deviation \\
\hline Age (years) & $65.44 \pm 12.85$ \\
\hline Male : Female & $19: 32$ \\
\hline Time to postoperative examination (months) & $3.63 \pm 3.43$ \\
\hline Target Spherical equivalent (diopter) & $-1.02 \pm 1.21$ \\
\hline Postoperative spherical equivalent (diopter) & $-1.17 \pm 1.44$ \\
\hline Postoperative cylinder (diopter) & $0.53 \pm 0.64$ \\
\hline Absolute prediction error (diopter) & $0.16 \pm 0.47$ \\
\hline Uncorrected visual acuity (LogMAR) & $0.27 \pm 0.28$ \\
\hline Best corrected visual acuity (LogMAR) & $0.04 \pm 0.07$
\end{tabular}

LogMAR: logarithm of the minimal angle of resolution

TABLE 2. Simulated best optical quality and matched defocus for the best area under the modulation transfer function curve after full correction of spherical aberration

\begin{tabular}{c|c|c|c|c}
\hline \hline \multirow{2}{*}{} & With innate SA & \begin{tabular}{c}
$\mid c$ \\
Total group \\
\cline { 3 - 5 }
\end{tabular} & & \multicolumn{2}{|c}{$\begin{array}{c}\text { Group 1 correction of SA } \\
(\mathrm{n}=47)\end{array}$} & $\begin{array}{c}\text { Group 2 } \\
(\mathrm{n}=17)\end{array}$ \\
\hline Defocus (diopter, D) & $-0.252 \pm 0.660$ & $0.083 \pm 0.528$ & $0.151 \pm 0.429$ & $-0.106 \pm 0.718$ \\
\hline aMTF & $6.614 \pm 2.164$ & $7.644 \pm 2.629$ & $8.210 \pm 2.489 *$ & $6.079 \pm 2.424 *$ \\
\hline
\end{tabular}

$* P$ value $<0.05$, repeated ANOVA

SA: spherical aberration

aMTF: area under modulation transfer function curve

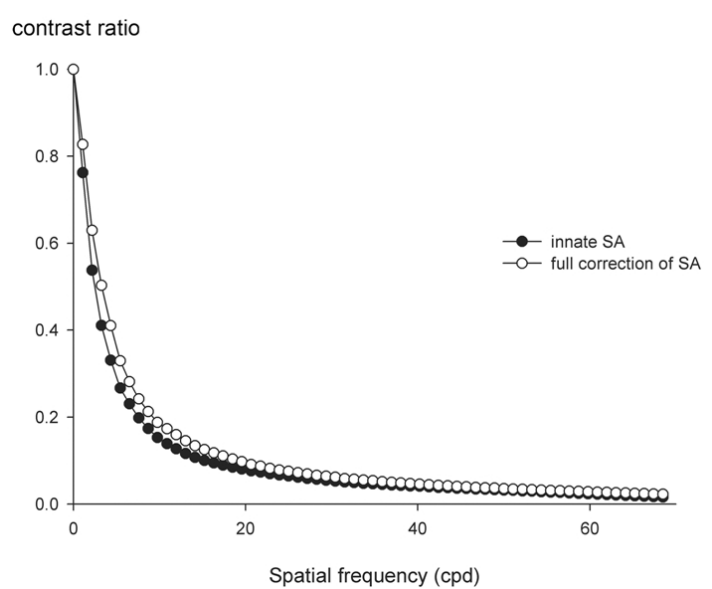

(a)

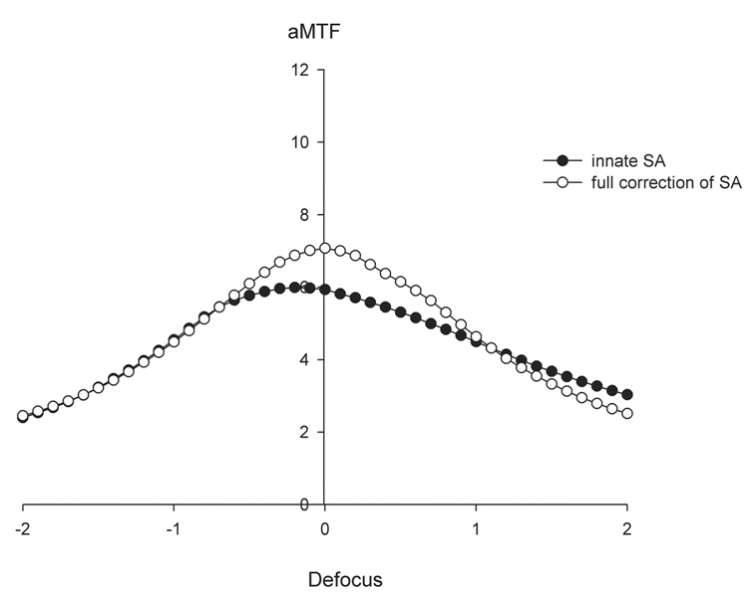

(b)

FIG. 3. Radially averaged MTF curve and the aMTF before and after full correction of postoperative SA.

These patients had a mean hyperopic shift of $0.40 \pm 0.63$ $\mathrm{D}$ (from $-0.252 \pm 0.660 \mathrm{D}$ to $0.151 \pm 0.429 \mathrm{D}$ ) and an average $22.4 \%$ increase of the aMTF (from $6.614 \pm 2.164$ to $8.210 \pm 2.489$, Table 2).

Table 3 shows the root mean square (RMS) of postoperative aberration in micrometers for all eyes, eyes with increased aMTF (Group 1), and eyes with decreased aMTF (Group 2) after full correction of innate SA. Group 2 had statistically significant differences at $Z(3,-1)$ (vertical coma, $p=0.01$ ) and $Z(4,4)$ (tetrafoil, $p=0.04$ ). Group 1 had slightly more SA than Group 2, but this was not statistically significant $(0.25 \pm 0.15$ vs. $0.19 \pm 0.10, p=0.19)$.

Figure 4 shows that the average of maximal aMTF through the range of defocus at each spherical aberration. For all eyes and Group 1, the maximal aMTF was at a SA of $0.02 \mu \mathrm{m}$ and $0.01 \mu \mathrm{m}$, respectively (aMTF $=7.66 \pm 2.69$ 
TABLE 3. Absolute values of postoperative aberration ( $\mu \mathrm{m}, 5 \mathrm{~mm}$ pupil size) in eyes (Group $1, \mathrm{n}=47$ ) with increased area under modulation transfer function curve (aMTF) and eyes with decreased aMTF (Group 2, n=17) after full correction of innate spherical aberration

\begin{tabular}{c|c|c|c|c}
\hline \hline & $\begin{array}{c}\text { Total group } \\
(\mathrm{n}=64)\end{array}$ & $\begin{array}{c}\text { Group 1 } \\
(\mathrm{n}=47)\end{array}$ & $\begin{array}{c}\text { Group 2 } \\
(\mathrm{n}=17)\end{array}$ & $P$ value \\
\hline RMS & $1.84 \pm 1.23$ & $1.65 \pm 1.07$ & $2.36 \pm 1.49$ & 0.09 \\
\hline RMS HOA & $0.53 \pm 0.38$ & $0.50 \pm 0.38$ & $0.61 \pm 0.39$ & 0.25 \\
\hline RMS w/o Z(4,0) & $0.47 \pm 0.40$ & $0.43 \pm 0.39$ & $0.56 \pm 0.41$ & 0.11 \\
\hline $3^{\text {rd }}$ RMS & $0.23 \pm 0.17$ & $0.21 \pm 0.16$ & $0.28 \pm 0.19$ & 0.13 \\
\hline $4^{\text {th }}$ RMS & $0.14 \pm 0.07$ & $0.13 \pm 0.07$ & $0.14 \pm 0.08$ & 0.92 \\
\hline $5^{\text {th }}$ RMS & $0.03 \pm 0.04$ & $0.03 \pm 0.03$ & $0.04 \pm 0.05$ & 0.61 \\
\hline$Z(3,-3)$ & $0.23 \pm 0.22$ & $0.22 \pm 0.24$ & $0.24 \pm 0.15$ & 0.46 \\
\hline$Z(3,-1)^{*}$ & $0.19 \pm 0.19$ & $0.16 \pm 0.17$ & $0.28 \pm 0.21$ & 0.01 \\
\hline$Z(3,1)$ & $0.15 \pm 0.14$ & $0.14 \pm 0.14$ & $0.18 \pm 0.15$ & 0.21 \\
\hline$Z(3,3)$ & $0.19 \pm 0.26$ & $0.17 \pm 0.21$ & $0.25 \pm 0.38$ & 0.81 \\
\hline$Z(4,-4)$ & $0.07 \pm 0.07$ & $0.07 \pm 0.07$ & $0.08 \pm 0.09$ & 0.89 \\
\hline$Z(4,-2)$ & $0.03 \pm 0.05$ & $0.03 \pm 0.03$ & $0.05 \pm 0.08$ & 0.77 \\
\hline$Z(4,0)$ & $0.23 \pm 0.14$ & $0.25 \pm 0.15$ & $0.19 \pm 0.10$ & 0.19 \\
\hline$Z(4,2)$ & $0.08 \pm 0.08$ & $0.07 \pm 0.07$ & $0.11 \pm 0.10$ & 0.08 \\
\hline$Z(4,4)^{*}$ & $0.09 \pm 0.10$ & $0.07 \pm 0.07$ & $0.14 \pm 0.16$ & 0.04 \\
\hline$Z(5,-5)$ & $0.03 \pm 0.04$ & $0.03 \pm 0.04$ & $0.04 \pm 0.05$ & 0.38 \\
\hline$Z(5,-3)$ & $0.03 \pm 0.03$ & $0.03 \pm 0.03$ & $0.02 \pm 0.03$ & 0.48 \\
\hline$Z(5,-1)$ & $0.03 \pm 0.04$ & $0.03 \pm 0.04$ & $0.03 \pm 0.04$ & 0.76 \\
\hline$Z(5,1)$ & $0.02 \pm 0.04$ & $0.02 \pm 0.02$ & $0.03 \pm 0.07$ & 0.95 \\
\hline$Z(5,3)$ & $0.03 \pm 0.04$ & $0.03 \pm 0.04$ & $0.03 \pm 0.03$ & 0.58 \\
\hline$Z(5,5)$ & $0.03 \pm 0.06$ & $0.03 \pm 0.04$ & $0.05 \pm 0.10$ & 0.53 \\
\hline
\end{tabular}

*: $P$-value $<0.05$, Mann-Whitney $\mathrm{U}$ test

aMTF: area under modulation transfer function curve

RMS: root mean square

RMS HOA: root mean square of higher-order aberration

RMS w/o Z $(4,0)$ : root mean square of higher-order aberration without spherical aberration

$3^{\text {rd }}$ RMS: root mean square of $3^{\text {rd }}$ order aberration

$4^{\text {th }}$ RMS: root mean square of $4^{\text {th }}$ order aberration

$5^{\text {th }}$ RMS: root mean square of $5^{\text {th }}$ order aberration

Z: coefficient of Zernike polynomial

and $8.22 \pm 2.54$, respectively). However, Group 2 had a maximal aMTF at a SA of $0.13 \mu \mathrm{m}(\mathrm{aMTF}=6.49 \pm 2.63)$.

\section{DISCUSSION}

In the present study, we investigated optical improvement after correction of SA under a relatively realistic postoperative situation. We simulated postoperative wavefront aberrations of eyes through the use of spherical IOLs in which there were no preoperative problems or intraoperative/postoperative complications (e.g., clinically detectable tilt or decentration of IOL) which could offset the possible benefits of an aspheric IOL $[3,4,22,23]$. In a previous pilot study (not published), we measured HOAs of IOLs using an optical bench, and found that the HOAs of the AR40e IOL was almost zero. Therefore, we simulated the postoperative HOAs of our eyes with the AR40e IOLs. Aberration-free IOLs are known to be less sensitive to tilt and decentration, which might minimize the HOAs induced by the IOL itself $[3,24]$.

Our results indicate that optical performance as a function of spatial frequency increased after full correction of innate SA at all spatial frequencies (Fig. 3). One of the strengths of this study is that objective measurements allowed us to divide subjects into two groups: those with increased aMTF, 
and those with decreased aMTF. Most previous studies on the benefits of aspheric IOLs involved comparisons between spherical IOLs and aspheric IOLs or a customized selection of various aspheric IOLs [4, 6, 10, 12-14, 25-28]. Some studies noted that the benefits from aspheric IOLs may be limited or diminished by inter-subject variability and interaction between HOAs [12, 16, 23]. However, no previous studies have established conditions that actually worsen optical performance. Our results indicate statistically significant differences between eyes with increased aMTF and those with decreased aMTF in the vertical coma and tetrafoil (Table 3). Previous studies found that corneal SA and coma did not change significantly with corneal incision and that other HOAs also did not change, especially after micro-incision cataract surgery [15, 29]. Therefore, it could be assumed that relatively high preoperative corneal HOA might be a negative predictive factor of optical outcomes using aspheric IOLs.

On the other hand, there are differences of opinion regarding the optimal postoperative SA. Our results indicate that a postoperative SA near zero appears to provide the best optical performance (Fig. 4). This result is supported by previous studies that used customized correction of SA to improve optical quality $[5,13,25,30]$. However, other previous clinical and experimental studies suggested that other targets for postoperative SA provide the best optical quality $[10,12,15]$. Nonetheless, the present study has some advantages over these other previous studies. We simulated optical quality with postoperative wavefront data and an AR40e IOL that had an SA of almost zero, which could more closely mimic the actual clinical situation. Moreover, the interval of our simulated SA was $0.01 \mu \mathrm{m}$, which presumably provides improved accuracy.

The eyes with decreased aMTF had slightly higher optical quality at an SA of $+0.13 \mu \mathrm{m}$ through the range of SAs. The optical quality was not totally concordant with the reduction of SA in Group 2. This might be due to an interaction of HOAs [16]. Thus, use of customized full correction of SA may improve optical quality, but a small residual SA might provide more benefits over full correction, especially for patients with high values of radially asymmetric optical components such as vertical coma.

Based on our results, the analysis of preoperative corneal aberration would be helpful in using aspheric or aberration free IOLs to improve optical outcomes. Though one of the considerations was the possibility of surgically induced HOAs, previous studies indicated that recent micro-incision cataract surgery led to no or minimal change of surgically induced HOAs [9, 29, 31]. This was why we attempted to simulate the postoperative ocular aberrations as the most clinically relevant condition instead of the preoperative corneal aberrations calculated on the basis of corneal topography. Another consideration is that we only included patients with AR40e IOLs who had uneventful implantations and no postoperative complications. In clinical practice, this might not exclude all influences in postoperative ocular

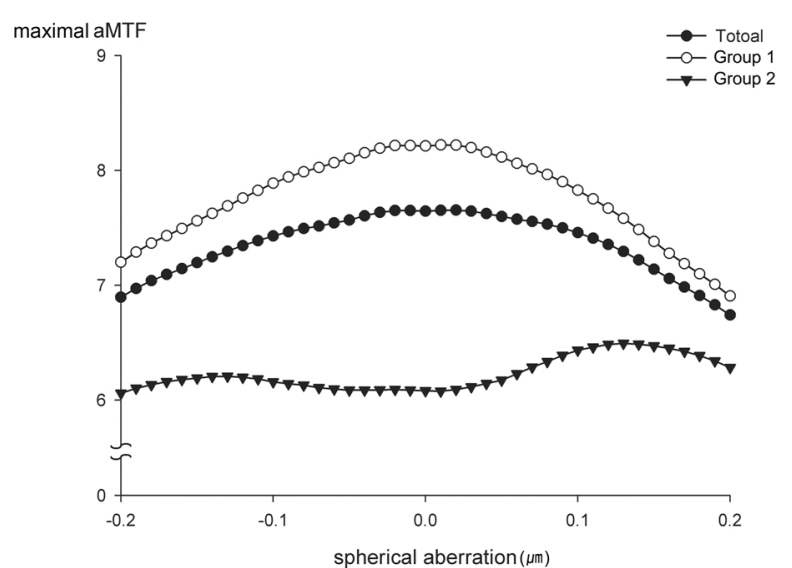

FIG. 4. Maximal value of the aMTF through the range of defocus at each SA.

aberrations of the IOL. However, aberration-free IOLs are known to be less sensitive to tilt and decentration, which might minimize the HOAs induced by the IOL itself, which was another reason why we attempted to simulate postoperative ocular aberrations as the most clinically relevant condition [3, 24].

\section{CONCLUSION}

To our knowledge, this study is the first report to demonstrate the optimal SA by use of simulated postoperative clinical wavefront data and to identify characteristics of eyes that might have some worsening of optical quality after full correction of SA. We revealed that seventeen eyes (26.6 $\%$ ) had decreased aMTF after full correction of the SA and statistically significant differences at $\mathrm{Z}(3,-1)$ (vertical coma, $p=0.01$ ) and $Z(4,4)$ (tetrafoil, $p=0.04$ ). Also, they had the maximal aMTF at a SA of $0.13 \mu \mathrm{m}$ compared with the maximal aMTF at a SA of $0.01 \mu \mathrm{m}$ in patients with improved aMTF after full correction of the SA. We suggest that future investigations examine the possible worsening of optical qualities after full correction of postoperative SA.

\section{ACKNOWLEDGMENT}

This work was partially supported by a grant (2010-400) from the Asan Institute for Life Sciences, Seoul, Korea. This work was partially supported by the National Research Foundation of Korea Grant funded by the Korean Government (NRF- 2014R1A1A2A16051562).

\section{REFERENCES}

1. J. Liang, B. Grimm, S. Goelz, and J. F. Bille, "Objective 
measurement of wave aberrations of the human eye with the use of a Hartmann-Shack wave-front sensor," J. Opt. Soc. Am. A Opt. Image Sci. Vis. 11, 1949-1957 (1994).

2. L. N. Thibos and X. Hong, "Clinical applications of the Shack-Hartmann aberrometer," Optom. Vis. Sci. 76, 817-825 (1999).

3. T. Eppig, K. Scholz, A. Loffler, A. Messner, and A. Langenbucher, "Effect of decentration and tilt on the image quality of aspheric intraocular lens designs in a model eye,” J. Cataract Refract. Surg. 35, 1091-1100 (2009).

4. R. Bellucci and S. Morselli, "Optimizing higher-order aberrations with intraocular lens technology," Curr. Opin. Ophthalmol. 18, 67-73 (2007).

5. J. T. Holladay, P. A. Piers, G. Koranyi, M. van der Mooren, and N. E. Norrby, "A new intraocular lens design to reduce spherical aberration of pseudophakic eyes," J. Refract. Surg. 18, 683-691 (2002).

6. R. Montes-Mico, T. Ferrer-Blasco, and A. Cervino, "Analysis of the possible benefits of aspheric intraocular lenses: review of the literature," J. Cataract Refract. Surg. 35, 172-181 (2009).

7. R. Navarro, L. Gonzalez, and J. L. Hernandez, "Optics of the average normal cornea from general and canonical representations of its surface topography," J. Opt. Soc. Am. A Opt. Image Sci. Vis. 23, 219-232 (2006).

8. M. K. Smolek and S. D. Klyce, "Goodness-of-prediction of Zernike polynomial fitting to corneal surfaces," J. Cataract Refract. Surg. 31, 2350-2355 (2005).

9. K. Negishi, C. Kodama, T. Yamaguchi, H. Torii, M. Saiki, M. Dogru, K. Ohnuma, and K. Tsubota, "Predictability of ocular spherical aberration after cataract surgery determined using preoperative corneal spherical aberration," J. Cataract Refract. Surg. 36, 756-761 (2010).

10. G. H. Beiko, "Personalized correction of spherical aberration in cataract surgery," J. Cataract Refract. Surg. 33, 14551460 (2007).

11. T. Yamaguchi, K. Negishi, T. Ono, H. Torii, M. Dogru, K. Yamaguchi, K. Ohnuma, and K. Tsubota, "Feasibility of spherical aberration correction with aspheric intraocular lenses in cataract surgery based on individual pupil diameter," J. Cataract Refract. Surg. 35, 1725-1733 (2009).

12. D. D. Koch and L. Wang, "Custom optimization of intraocular lens asphericity," Trans. Am. Ophthalmol. Soc. 105, 36-41; discussion 41-32 (2007).

13. M. Packer, I. H. Fine, and R. S. Hoffman, "Aspheric intraocular lens selection based on corneal wavefront," J. Refract Surg. 25, 12-20 (2009).

14. P. A. Piers, E. J. Fernandez, S. Manzanera, S. Norrby, and P. Artal, "Adaptive optics simulation of intraocular lenses with modified spherical aberration," Invest. Ophthalmol. Vis Sci. 45, 4601-4610 (2004).

15. Y. Nochez, A. Favard, S. Majzoub, and P. J. Pisella, "Measurement of corneal aberrations for customisation of intraocular lens asphericity: impact on quality of vision after micro-incision cataract surgery," Br. J. Ophthalmol. 94, 440-444 (2009).

16. R. A. Applegate, J .D. Marsack, R. Ramos, and E. J. Sarver,
"Interaction between aberrations to improve or reduce visual performance," J. Cataract Refract Surg. 29, 1487-1495 (2003).

17. P. M. Prieto, F. Vargas-Martin, S. Goelz, and P. Artal, "Analysis of the performance of the Hartmann-Shack sensor in the human eye," J. Opt. Soc. Am. A Opt. Image Sci. Vis. 17, 1388-1398 (2000).

18. G. Y. Yoon and D. R. Williams, "Visual performance after correcting the monochromatic and chromatic aberrations of the eye," J. Opt. Soc. Am. A Opt. Image Sci. Vis. 19, 266-275 (2002).

19. H. Kobashi, K. Kamiya, K. Yanome, A. Igarashi, and K. Shimizu, "Effect of pupil size on optical quality parameters in astigmatic eyes using a double-pass instrument," Biomed. Res. Int. 2013, 124327 (2013).

20. K. Y. Li and G. Yoon, "Changes in aberrations and retinal image quality due to tear film dynamics," Opt. Express 14, 12552-12559 (2006).

21. J. DeCoster, "Testing group differences using t-tests, ANOVA, and nonparametric measures," (2006).

22. L. Wang and D. D. Koch, "Effect of decentration of wavefront-corrected intraocular lenses on the higher-order aberrations of the eye," Arch. Ophthalmol. 123, 1226-1230 (2005).

23. H. H. Dietze and M. J. Cox, "Limitations of correcting spherical aberration with aspheric intraocular lenses," J. Refract. Surg. 21, S541-546 (2005).

24. F. Taketani, T. Matuura, E. Yukawa, and Y. Hara, "Influence of intraocular lens tilt and decentration on wavefront aberrations," J. Cataract Refract. Surg. 30, 2158-2162 (2004).

25. J. D. Solomon, "Outcomes of corneal spherical aberration-guided cataract surgery measured by the OPD-scan," J. Refract. Surg. 26, 863-869 (2010).

26. G. H. Beiko, W. Haigis, and A. Steinmueller, "Distribution of corneal spherical aberration in a comprehensive ophthalmology practice and whether keratometry can predict aberration values," J. Cataract Refract. Surg. 33, 848-858 (2007).

27. M. A. Nanavaty, D. J. Spalton, J. Boyce, S. Saha, and J. Marshall, "Wavefront aberrations, depth of focus, and contrast sensitivity with aspheric and spherical intraocular lenses: fellow-eye study," J. Cataract Refract. Surg. 35, 663-671 (2009).

28. K. W. van Gaalen, S. A. Koopmans, N. M. Jansonius, and A. C. Kooijman, "Clinical comparison of the optical performance of aspheric and spherical intraocular lenses," J. Cataract Refract. Surg. 36, 34-43 (2010).

29. S. Marcos, P. Rosales, L. Llorente, and I. Jimenez-Alfaro, "Change in corneal aberrations after cataract surgery with 2 types of aspherical intraocular lenses," J. Cataract Refract. Surg. 33, 217-226 (2007).

30. S. Marcos, S. Barbero, and I. Jimenez-Alfaro, "Optical quality and depth-of-field of eyes implanted with spherical and aspheric intraocular lenses," J. Refract Surg. 21, 223-235 (2005).

31. A. Guirao, J. Tejedor, and P. Artal, "Corneal aberrations before and after small-incision cataract surgery," Invest. Ophthalmol. Vis. Sci. 45, $4312-4319$ (2004). 